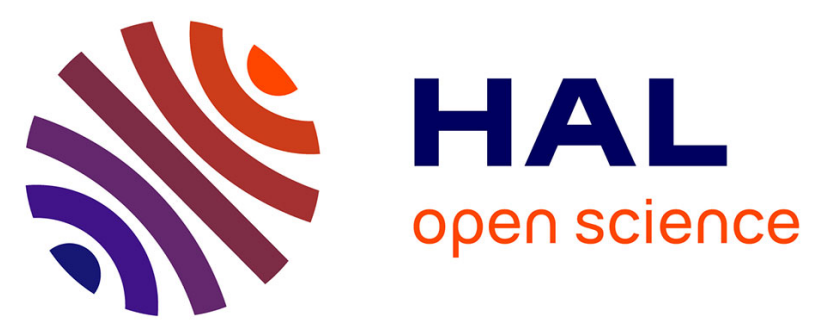

\title{
Efficient photonic mirrors for semiconductor nanowires
}

Inbal Friedler, Philippe Lalanne, Jean-Paul Hugonin, Julien Claudon, Jean-Michel Gérard, Alexios Beveratos, Isabelle Robert-Philip

\section{To cite this version:}

Inbal Friedler, Philippe Lalanne, Jean-Paul Hugonin, Julien Claudon, Jean-Michel Gérard, et al.. Efficient photonic mirrors for semiconductor nanowires. Optics Letters, 2008, 33 (22), pp.2635-2637. hal-00566665

\section{HAL Id: hal-00566665 \\ https://hal-iogs.archives-ouvertes.fr/hal-00566665}

Submitted on 5 Apr 2012

HAL is a multi-disciplinary open access archive for the deposit and dissemination of scientific research documents, whether they are published or not. The documents may come from teaching and research institutions in France or abroad, or from public or private research centers.
L'archive ouverte pluridisciplinaire HAL, est destinée au dépôt et à la diffusion de documents scientifiques de niveau recherche, publiés ou non, émanant des établissements d'enseignement et de recherche français ou étrangers, des laboratoires publics ou privés. 


\title{
Efficient photonic mirrors for semiconductor nanowires
}

\author{
I. Friedler, ${ }^{1}$ P. Lalanne, ${ }^{1, *}$ J. P. Hugonin, ${ }^{1}$ J. Claudon, ${ }^{2}$ J. M. Gérard,${ }^{2}$ A. Beveratos, ${ }^{3}$ and I. Robert-Philip ${ }^{3}$ \\ ${ }^{1}$ Laboratoire Charles Fabry de l'Institut d'Optique, CNRS, Universite Paris-Sud, Campus Polytechnique, RD 128, \\ 91127 Palaiseau, France \\ ${ }^{2}$ Commissariat a l'Energie Atomique (CEA)/INAC/SP2M, Nanophysics and Semiconductor Laboratory, \\ 17 Rue des Martyrs, 38054 Grenoble Cedex 9, France \\ ${ }^{3}$ Laboratoire de Photonique et de Nanostructures, CNRS, Route de Nozay, 91460 Marcoussis Cedex, France \\ *Corresponding author: philippe.lalanne@institutoptique.fr
}

Received August 25, 2008; revised September 29, 2008; accepted October 1, 2008; posted October 15, 2008 (Doc. ID 100618); published November 12, 2008

Using a fully vectorial frequency-domain aperiodic Fourier modal method, we study nanowire metallic mirrors and their photonic performance. We show that the performance of standard quarter-wave Bragg mirrors at subwavelength diameters is surprisingly poor, while engineered metallic mirrors that incorporate a thin dielectric adlayer may offer reflectance larger than $90 \%$ even for diameters as small as $\lambda / 5$. () 2008 Optical Society of America

OCIS codes: 130.2790, 350.4238, 260.3910, 250.5590.

There is much current interest in the optical properties of single-crystal nanowires, since their strong $2 \mathrm{D}$ confinement of electrons, holes, and photons make them particularly attractive as potential building blocks for nanoscale electronics and optoelectronic devices, including lasers [1-3], light detection in photovoltaic devices [4], ultrasmall optical waveguides [5], and single-photon sources [6,7]. As shown by optical and electrical measurements, semiconductor nanowires can function as Fabry-Perot optical cavities with mode spacing inversely related to the nanowire length. Thus, the modal reflectance at the wire termination (the facets for cleaved nanowires for instance) drastically impacts the photonic performance, such as the threshold current for lasers or the $Q$ factor for nanocavities [3], or even more basically, it may preempt radiation into an undesired facet and reinforce the directivity of the emitted light. Thus the realization of nanowire mirrors with high reflectance becomes crucial for applications.

In this Letter, we theoretically study the reflectivity of GaAs nanowire facets for different kinds of mirrors. We first show that standard Bragg mirrors are rather inefficient for wire diameters $D<\lambda / 3$. By contrast, we show that a high reflectance can be achieved for properly designed metallic mirrors. We consider both nanowires standing on a planar reflector and nanowires terminated by a metallic post. For the first geometry, the reflectance exhibits a pronounced dip within the diameter range for singlemode operation, due to the excitation of surface plasmon polaritons (SPPs). This effect can be quenched by incorporating a thin dielectric spacer layer between the GaAs wire and metallic mirror, and a high reflectance $(R>90 \%)$ can be achieved for all singlemode wires. The second geometry, which is attractive due to the compactness of integrated post-mirrors, is not as efficient for small diameters, but offers a rather large reflectance $(R>80 \%)$ for $D>0.23 \lambda$.

In the following, we consider cylindrical nanowires made of high refractive index semiconductors such as
GaAs $(n=3.45)$. The wires offer a strong $2 \mathrm{D}$ confinement of light in the transverse directions and guide light in the longitudinal $z$ direction. Figure 1(a) shows the normalized propagation constant $n_{\text {eff }}$ $=k_{z} / k_{0}$ for the fundamental $\mathrm{HE}_{11}$ mode [8] as a function of $D / \lambda$. The computational results are obtained at $\lambda \gamma=950 \mathrm{~nm}$, a wavelength corresponding to the emission line of single InAs quantum dots [7], with a fully vectorial frequency-domain aperiodic Fourier modal method (a-FMM) [9]. The overall dispersion of the guided mode can be separated into three representative regimes. For small diameters $(D<0.18 \lambda)$, $n_{\text {eff }}$ is close to unity and the mode band is very close to the light line of the background free space. In this
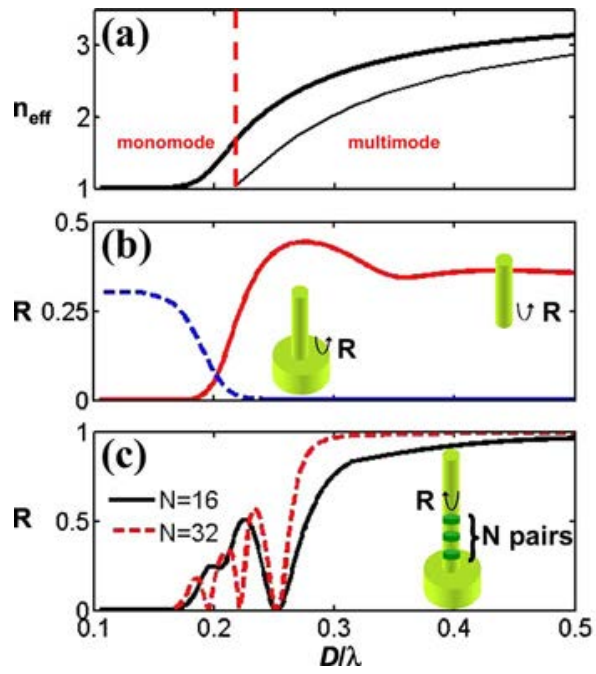

Fig. 1. (Color online) (a) Effective index $n_{\text {eff }}$ of the $\mathrm{HE}_{11}$ mode of a GaAs cylindrical nanowire as a function of the normalized diameter $D / \lambda$. The second mode is shown with a thin curve. (b) $\mathrm{HE}_{11}$ modal reflectance of a cleaved facet in air (solid curve) and of a facet connecting with a GaAs substrate (dashed curve). (c) $\mathrm{HE}_{11}$ modal reflectance of an $N$-pair GaAs/AlAs $(n=3.45 / 2.95)$ Bragg mirror composed of $\lambda /\left(4 n_{\text {eff }}\right)$ thick layers for $N=16$ (solid curve) and $N=32$ (dashed curve). All results hold for $\lambda=950 \mathrm{~nm}$. 
domain, the field is only weakly confined and a large fraction of the mode power witnesses the air background. For $D>0.3 \lambda, n_{\text {eff }}$ is rather large, implying that the $\mathrm{HE}_{11}$ field is almost entirely confined within the nanowire. Between the weak- and strongconfinement regimes lays an intermediate domain where the dispersion significantly deviates from the light lines of the air and GaAs materials. This represents the interesting regime for most photonic applications; monomode operation is guaranteed but more importantly, a large fraction of the spontaneous emission of a dipole (a quantum dot for instance) embedded in the wire is weakly coupled to radiation modes and preferentially excites the fundamental $\mathrm{HE}_{11}$ mode $[7,10]$. This property may have important consequences for the design and performance of broadband single-photon sources $[7,11]$.

Let us first consider standard nanowire terminations. Figure 1(b) shows the reflectivity of the fundamental $\mathrm{HE}_{11}$ mode for a cleaved facet in air (solid red curve) and for a facet connected to a GaAs substrate (dashed blue curve). The two curves show opposite behaviors that simply reflect the variation of the field confinement as $D / \lambda$ varies. In our search for better performance, we further study Bragg mirrors, as suggested in the context of nanowire lasers [12]. The a-FMM calculations have been performed for the GaAs/AlAs system, which is well-mastered technologically and which has recently allowed the observation of $Q$ factors of $\approx 1000$ for micropillars with diameters in the range of 500-600 nm [13]. Figure 1(c) shows the reflectance of GaAs/AlAs $(n=3.45 / 2.95)$ Bragg mirrors composed of $N=16$ and 32 pairs and connected to the GaAs substrate. For the calculation performed at a fixed wavelength $(\lambda=950 \mathrm{~nm})$, each layer of the reflector is assumed to have a quarterwave thickness $\left(\lambda /\left[4 n_{\text {eff }}\right]\right)$ for every $D$ implying that, as $D$ varies, the layer thicknesses also vary. As shown in Fig. 1(c), the Bragg-mirror reflectivity remains small for $D<0.3 \lambda$. It is noteworthy that it is nearly null for $D=0.25 \lambda$ and for $N=16$ and 32 . As shown by further computations, this is due to the very weak $(<0.2 \%) \mathrm{HE}_{11}$ modal reflectance at GaAs/AlAs interface for this diameter. We additionally note that, because of the weak refractive index contrast for small $D$ s, a large number of alternate layers is required for achieving a moderate reflectivity. This is challenging for fabrication.

Pursuing alternative ways to achieve a high reflectivity we next study facets connecting to a metallic silver mirror [inset in Fig. 2(a)]. Hereafter, silver is considered as a real metal with a finite permittivity [14], $\varepsilon=-41+2.5 i$ at $\lambda=950 \mathrm{~nm}$. The solid blue curve in Fig. 2(a) shows the mirror reflectivity, as a function of the normalized wire diameter. It exhibits a pronounced dip, $R=0.3 \%$ for $D=0.19 \lambda$. Figure 2(b) shows the radial component of the magnetic-field vector under illumination by the fundamental $\mathrm{HE}_{11}$ mode for $D=0.19 \lambda$. As shown, the low reflectance is due to a self-focusing effect at the metallic facet, where $15 \%$ of the incoming light is absorbed just below the wire in silver. To further understand the rea-
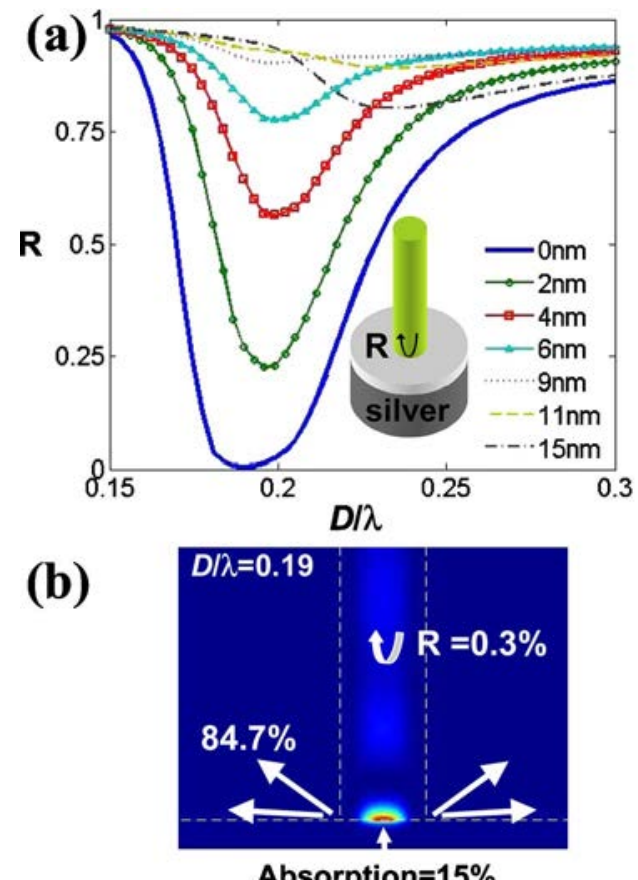

Absorption $=15 \%$

Fig. 2. (Color online) Performance of facets connecting with a silver substrate. (a) $\mathrm{HE}_{11}$ modal reflectance on a planar silver mirror either bare $(t=0$, solid curve) or covered by a thin dielectric adlayer $(n=1.5)$ of thickness $t$ $(\lambda=950 \mathrm{~nm})$ (b) Longitudinal cross section for the $y$ component of the magnetic-field pattern for $D / \lambda=0.19$ and $t=0$ $(R=0.3 \%)$, showing a self-focusing effect at the nanowiresilver interface, where $15 \%$ of the incident $\mathrm{HE}_{11}$ energy is absorbed.

son for this surprising phenomenon, we have performed $2 \mathrm{D}$ calculations for GaAs slab waveguides. We found that the dip only exists for TM polarization (magnetic-field vector parallel to the slab), but not for TE polarized light (electric-field vector parallel to the slab). Inspection of the field pattern at minimum reflectance $(R=0.086)$ has revealed a self-focusing effect similar to that reported in Fig. 2(b), with $14 \%$ of the incoming light that is locally absorbed just below the slab in silver. For the 2D slab geometry it is possible to rigorously calculate [15] the excitation efficiencies of the SPPs launched on both side of the GaAs slab. We found that this heat channel represents $32 \%$ of the incident light and that the remaining $46 \%$ are backscattered into air. As suspected, the self-focusing effect is accompanied by an efficient excitation of SPPs. We believe that this also holds for the 3D cylindrical wire geometry in Fig. 2(b).

To suppress the plasmonic excitation we suggest coating the silver substrate with a thin oxide adlayer $(n=1.5)$. The results are shown in Fig. 2(a). The best performance $(R>90 \%)$ over a broad range of diameters is achieved for thicknesses of $t=5-10 \mathrm{~nm}$. The oxide adlayer indeed reduces the plasmonic excitation and the metallic absorption. We have also varied the refractive index of the adlayer from $n=1.5$ to 3.5. A high reflectance has been systematically obtained by optimizing the adlayer thickness.

We now consider the metallic post mirror geometry sketched in the inset of Fig. 3. Such mirrors might 


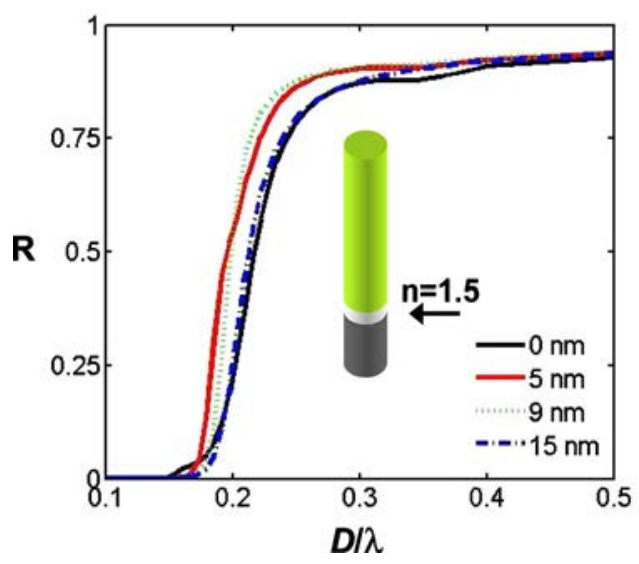

Fig. 3. (Color online) $\mathrm{HE}_{11}$ modal reflectance of facets connecting with a silver post (inset) for several values of the adlayer $(n=1.5)$ thickness $t=0,5,9$, and $15 \mathrm{~nm}(\lambda$ $=950 \mathrm{~nm}$ ).

prove more compact and versatile than planar ones. Figure 3 shows the facet reflectance as a function of $D / \lambda$ for several adlayer $(n=1.5)$ thicknesses. As expected, low reflectance is always observed for small diameters due to the weak interaction between the metallic post and the $\mathrm{HE}_{11}$ field that is weakly confined. However, interestingly, the post geometry offers high reflectance $(R>80 \%)$ for $D>0.26 \lambda$ (black solid curve), or even for $D>0.22 \lambda$ (dotted green curve) provided that a $9 \mathrm{~nm}$ thick adlayer is inserted at the GaAs-gold interface. For this optimal thickness, 0.9 reflectance is achieved for $D$ as small as $0.28 \lambda$. The computational results in Fig. 3 are obtained for a semi-infinite metal post, but virtually identical results have been obtained for $100 \mathrm{~nm}$ thick posts, letting us expect a similar reflectance for goldcoated facets in freestanding nanowires. We have also studied the performance of the planar and post mirror at $\lambda=1500 \mathrm{~nm}$ using either gold or silver. Results similar to those in Figs. 2 and 3 have been obtained for both metals.

We are currently investigating the planar-mirror fabrication by depositing the adlayer and the metal film onto a planar GaAs wafer incorporating a sacrificial GaAlAs layer. Then wafer bonding techniques are used to stick the sample upside down on another substrate, and the nanowire is finally processed by standard $e$-beam lithography and reactive ion etching. The post geometry can be further obtained by using an additional ion beam etching step. It can also be fabricated by direct thin film deposition onto a cleaved facet. Note that quite similar geometries are also obtained when gold droplets are used as catalysts to assist nanowire growths [16]. Such mirrors could improve the efficiency of single-photon sources or could be incorporated to reduce the laser threshold of elementary nanowire resonators, offering quality factors of $\sim 100$ for first-order Fabry-Perot resonances.

The authors gratefully acknowledge fruitful discussions with G. Lecamp and B. Gayral. The work has been performed under the NanoEPR project of the 2006 NanoSci-ERA European program.

\section{References}

1. X. F. Duan, Y. Huang, R. Agarwal, and C. M. Lieber, Nature (London) 421, 241 (2003).

2. J. C. Johnson, H. Q. Yan, P. D. Yang, and R. G. Saykally, J. Phys. Chem. B 107, 8816 (2003).

3. M. T. Hill, Y. S. Oei, E. Smalbrugge, Y. Zhu, T. De Vries, P. J. van Veldhoven, F. W. M. van Otten, T. J. Eijkemans, J. P. Turkiewicz, H. De Waardt, E. J. Geluk, S. H. Kwon, Y. H. Lee, R. Nötzel, and M. K. Smit, Nat. Photonics 1, 589 (2007).

4. B. Z. Tian, X. L. Zheng, T. J. Kempa, Y. Fang, N. F. Yu, G. H. Yu, J. L. Huang, and C. M. Lieber, Nature (London) 449, 885 (2007).

5. Y. K. Lize, E. C. Magi, V. G. Ta'eed, J. A. Bolger, P. Steinvurzel, and B. J. Eggleton, Opt. Express 12, 3209 (2004).

6. M. T. Borgström, V. Zwiller, E. Müller, and A. Imamoğlu, Nano Lett. 5, 1439 (2005).

7. Y. R. Nowicki-Bringuier, R. Hahner, J. Claudon, G. Lecamp, P. Lalanne, and J. M. Gérard, Ann. Phys. (Paris) 32, 151 (2008).

8. A. W. Snyder and J. D. Love, Optical Waveguide Theory (Chapman and Hall, 1983).

9. J. P. Hugonin and P. Lalanne, J. Opt. Soc. Am. A 22, 1844 (2005).

10. For a radial dipole, the fraction of the dipole emission that is coupled in the fundamental HE11 mode may exceed $90 \%$ for $D / \lambda=0.23$; see D. Y. Chu and S. T. Ho, J. Opt. Soc. Am. B 10, 381 (1993).

11. G. Lecamp, P. Lalanne, and J. P. Hugonin, Phys. Rev. Lett. 99, 023902 (2007).

12. L. Chen and E. Towe, Appl. Phys. Lett. 89, 053125 (2006).

13. G. Lecamp, J. P. Hugonin, P. Lalanne, R. Braive, S. Varoutsis, S. Laurent, A. Lemaître, I. Sagnes, G. Patriarche, I. Robert-Philip, and I. Abram, Appl. Phys. Lett. 90, 091120 (2007).

14. E. D. Palik, Handbook of Optical Constants of Solids (Academic, 1985), Part II(1).

15. P. Lalanne, J. P. Hugonin, and J. C. Rodier, J. Opt. Soc. Am. A 23, 1608 (2006)

16. P. J. Pauzauskie and P. Yang, Mater. Today 9, 36 (2006). 